\title{
Perturbative Reformulation of the Stochastic Galerkin Method for Statistical Analysis of Wiring Structures with Several Random Parameters
}

\begin{abstract}
In this paper, a novel approach for statistical analysis of cable harnesses characterized by several random parameters is proposed, which is based on a perturbative reformulation of the well-known Stochastic Galerkin Method (SGM). With respect to traditional SGM, the proposed method avoids the solution of an augmented multiconductor transmission line (MTL) whose dimensions may become prohibitive in case of structures characterized by several wires and random parameters. Namely, it resorts to iterative solution of MTLs with the same number of wires of the original structure, where effects due to random variation of geometrical parameters are included by induced sources. The proposed approach is here applied to collect statistical information of voltages and currents at the terminations of a shielded cable. Through such an example, involving a large number of wires (7) and random variables (12), it is proven that the proposed method allows a significant reduction of computational time with respect to traditional SGM, assuring the same accuracy in the prediction of statistical moments.
\end{abstract}

Index Terms-Multiconductor transmission lines, perturbation technique, shielded cable, statistical analysis, stochastic Galerkin method.

\section{INTRODUCTION}

Statistical techniques recently gained increasing attention from the Electromagnetic Compatibility (EMC) community, owing to the need to handle systems in which some parameters may randomly vary either due to manufacturing tolerances or due to uncertainty in the knowledge of their exact value. This is, for instance, the case of complex wiring harnesses, whose geometrical characteristics, such as precise position of wires in the bundle, are often hard to be controlled [1-4]. Since these random variations may significantly impact EMC performance, modeling and propagating the uncertainty associated with these parameters become of paramount importance in order to provide a statistical characterization (in terms of statistical moments) of relevant electrical quantities. Towards this goal, the standard approach, known as Monte Carlo (MC) method, is to perform repeated simulations exploiting different realizations of the system under analysis. However, in order to achieve accuracy in the prediction of statistical moments, a huge number of samples is required, and this can lead to unacceptable computational time, unless the underlying deterministic model is very simple. In order to overcome such a limitation without degrading prediction accuracy, more advanced statistical techniques have been recently proposed. Among these, the Stochastic Galerkin Method (SGM) offers characteristics particularly suited for accurate statistical as- sessment of cables and interconnects with geometry affected by uncertainty [5]. This method resorts to Polynomial Chaos Expansion (PCE) of the random quantities of the problem, that is per-unit-length (p.u.l.) parameters (input quantities) and line voltages and currents (output quantities). These quantities are expanded in series of orthogonal polynomials, and used to derive an augmented, but deterministic, model of the original system, whose solution directly provides statistical estimates of the output variables, without requiring repeated simulations. When applied to wiring structures characterized by a limited number of wires and/or random variables, this technique was proven to be very accurate and computationally efficient [6]. However, due to its intrusiveness, the computational burden of SGM becomes prohibitive when the number of random variables and/or conductors is very large. In order to overcome the aforesaid limitation, this paper proposes an SGM reformulation, which is obtained by combining the traditional SGM with the perturbative technique recently proposed in [7], [8] for the solution of nonuniform transmission lines. According to this novel approach, solution of the augmented multiconductor transmission line (MTL) model obtained by the SGM is avoided, by converting such an augmented network into subnetworks having the same number of wires of the original structure. The solution is achieved iteratively, by including effects due to random variability of geometrical parameters by distributed current and voltage sources. Accuracy and computational efficiency of this hybrid procedure is here investigated considering a shielded cable with random position of the inner wires $[9,10]$ as an application example.

\section{StRucture Under AnAlysis}

Without loss of generality, we consider in this work the shielded cable depicted in Fig. 1(a). The geometry was originally described in [9]. Later, this cable was stochastically studied in [10]. The cable consists of seven coated wires, with conductor radius $r_{c}$ and dielectric radius $r_{d}=2 r_{c}$. One wire is located at the center of the shield, whereas the remaining six wires lie symmetrically around the center at a radial distance of $d=5 r_{c}$. The relative permittivity of the dielectric coating $\varepsilon_{r}=4$. The shield radius is $r_{s h}=10 r_{c}{ }^{1}{ }^{1}$ The cable is 4-meter long and uniform along its length. Without loss of generality, in this work we consider a differential source

\footnotetext{
${ }^{1}$ Since only relative dimensions count, the value of $r_{c}$ is arbitrary.
} 


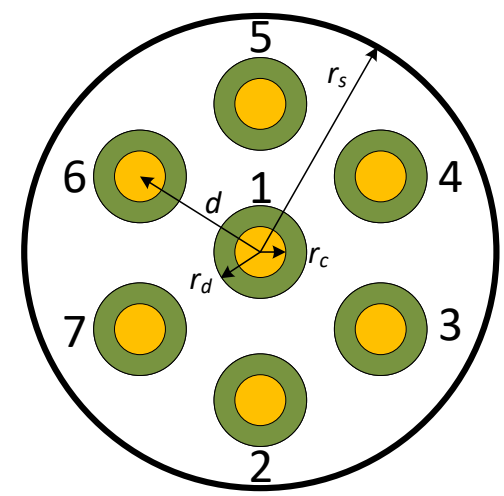

(a)

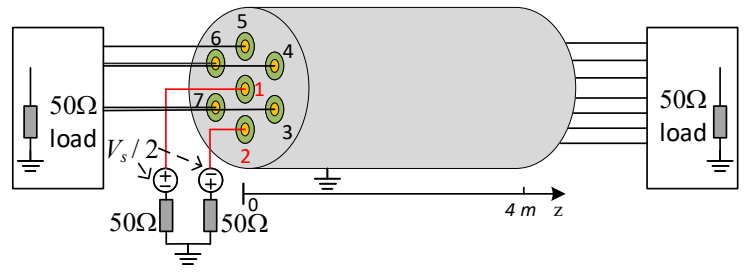

(b)

Fig. 1: Cross-section of the shielded cable under analysis (a); cable configuration with terminations (b).

connected between wires no. 1 and no. 2 , and 50- $\Omega$ loads connected to each wire as depicted in Fig. 1(b).

For the stochastic analysis, the positions of the outer wires (no. 2 to no. 7) are considered to be random. Namely, the horizontal and vertical coordinates of these wires are uniformly distributed within $\pm 10 \%$ around their nominal values. The total number of random variables is thus 12 .

\section{StATE-OF-The-ART GALERKIN APPROACH}

The frequency-domain equations for the cable under investigation read [11]:

$$
\begin{aligned}
\frac{d}{d z} \mathbf{V}(z, \omega, \boldsymbol{\xi}) & =-j \omega \mathbf{L}(\boldsymbol{\xi}) \mathbf{I}(z, \omega, \boldsymbol{\xi}), \\
\frac{d}{d z} \mathbf{I}(z, \omega, \boldsymbol{\xi}) & =-j \omega \mathbf{C}(\boldsymbol{\xi}) \mathbf{V}(z, \omega, \boldsymbol{\xi}),
\end{aligned}
$$

where $\omega$ is the angular frequency, and $\mathbf{L}$ and $\mathbf{C}$ are the pertinent p.u.l. inductance and capacitance matrices, respectively. Vectors $\mathbf{V}=\left[V_{1}, \ldots, V_{7}\right]^{T}$ and $\mathbf{I}=\left[I_{1}, \ldots, I_{7}\right]^{T}$ collect the line voltages and currents, respectively, whereas vector $\boldsymbol{\xi}=\left[\xi_{1}, \ldots, \xi_{12}\right]$ encompasses the random parameters (i.e., the wire positions). The p.u.l. inductance and capacitance matrices depend on $\boldsymbol{\xi}$ and they are therefore stochastic, as a result of the variability of the wire positions. Because of this randomness, wire voltages and currents are in turn also stochastic.

The classical MC approach collects statistical information by repeatedly solving (1) for multiple samples of the random parameters. However, the MC analysis is rather slow due to the non-negligible time required by the evaluation of the p.u.l. parameters, and by the analysis of the corresponding transmission-line configurations. In order to significantly speed-up the p.u.l. parameter extraction, a third-order PCE was calculated in [10] to statistically characterize the p.u.l. parameters:

$$
\begin{aligned}
\mathbf{L} & =\sum_{k=0}^{454} \mathbf{L}_{k} \varphi_{k}(\boldsymbol{\xi}) \\
\mathbf{C} & =\sum_{k=0}^{454} \mathbf{C}_{k} \varphi_{k}(\boldsymbol{\xi}),
\end{aligned}
$$

where the basis functions $\varphi_{k}$ are multivariate Legendre polynomials. From the PCE, relevant statistical information is readily derived [12]. The number of 455 PCE coefficients results from a third-order expansion with 12 random parameters.

Once the PCEs of p.u.l. parameters are obtained, equations (1) can be recast in terms of an augmented and deterministic MTL-like equations by means of the SGM [6]:

$$
\begin{aligned}
\frac{d}{d z} \tilde{\mathbf{V}}(z, \omega) & =-j \omega \tilde{\mathbf{L}} \tilde{\mathbf{I}}(z, \omega), \\
\frac{d}{d z} \tilde{\mathbf{I}}(z, \omega) & =-j \omega \tilde{\mathbf{C}} \tilde{\mathbf{V}}(z, \omega),
\end{aligned}
$$

where vectors $\tilde{\mathbf{V}}$ and $\tilde{\mathbf{I}}$ collect the PCE coefficients of the wire voltages and currents, whereas the new p.u.l. matrices $\tilde{\mathbf{L}}$ and $\tilde{\mathbf{C}}$ are formed by a suitable combination of the PCE coefficients in (2). By solving the deterministic MTL problem (3), a statistical characterization of such voltages and currents in terms of PCEs like (2) is obtained. Equation (3) is 455 times larger than (1), therefore being equivalent to a line with 3185 conductors instead of 7 ! This is the so-called "curse of dimensionality", which reduces the efficiency of the SGM for problems with high order and/or number of random parameters.

\section{Proposed Perturbative Galerkin Approach}

In order to alleviate the computational burden of the direct solution of (3) as a (rather huge) coupled MTL, a perturbation technique is applied in order to solve the SGM problem (3) in an iterative and decoupled manner. This perturbation technique is a reformulation of the one originally proposed in $[7,8]$ for the analysis of nonuniform MTLs.

The proposed approach starts by isolating the first component in the PCEs (2), which corresponds to the average value and is constant w.r.t. $\boldsymbol{\xi}$, being $\varphi_{0}(\boldsymbol{\xi})=1$ :

$$
\begin{aligned}
& \mathbf{L}=\mathbf{L}_{0}+\sum_{k=1}^{454} \mathbf{L}_{k} \varphi_{k}(\boldsymbol{\xi}) \\
& \mathbf{C}=\mathbf{C}_{0}+\sum_{k=1}^{454} \mathbf{C}_{k} \varphi_{k}(\boldsymbol{\xi})
\end{aligned}
$$


With this modification, the SGM produces

$$
\begin{aligned}
& \frac{d}{d z} \tilde{\mathbf{V}}(z, \omega)=-j \omega\left[\begin{array}{ccc}
\mathbf{L}_{0} & & \\
& \ddots & \\
& & \mathbf{L}_{0}
\end{array}\right] \tilde{\mathbf{I}}(z, \omega)-j \omega \widetilde{\boldsymbol{\Delta} \mathbf{L}} \tilde{\mathbf{I}}(z, \omega) \\
& \frac{d}{d z} \tilde{\mathbf{I}}(z, \omega)=-j \omega\left[\begin{array}{lll}
\mathbf{C}_{0} & & \\
& \ddots & \\
& & \mathbf{C}_{0}
\end{array}\right] \tilde{\mathbf{V}}(z, \omega)-j \omega \widetilde{\boldsymbol{\Delta} \mathbf{C}} \tilde{\mathbf{V}}(z, \omega),
\end{aligned}
$$

where the diagonal matrices in the r.h.s. are the result of the Galerkin projection of the deterministic zero-order components, whereas the augmented matrices $\widetilde{\Delta L}$ and $\widetilde{\Delta C}$ are conceptually identical to $\widetilde{\mathbf{L}}$ and $\widetilde{\mathbf{C}}$ in (3). Yet, they do not account for the aforementioned average contributions, but only for the random displacement therefrom.

It should be noted that the norms of $\widetilde{\Delta L}$ and $\widetilde{\Delta C}$ are expected to be (significantly) smaller than the norms of $\mathbf{L}_{0}$ and $\mathbf{C}_{0}$. This is because the expansions (2) are convergent, and the norms of their coefficients decay exponentially to zero [12]. This interpretation allows solving equation (5) iteratively by resorting to a perturbation approach $[7,8]$. By denoting with the subscript $m$ the solution at the $m$ th iteration step, equation (5) becomes

$$
\begin{aligned}
& \frac{d}{d z} \tilde{\mathbf{V}}_{m}(z, \omega) \\
& =-j \omega\left[\begin{array}{ccc}
\mathbf{L}_{0} & & \\
& \ddots & \\
& & \mathbf{L}_{0}
\end{array}\right] \tilde{\mathbf{I}}_{m}(z, \omega)-j \omega \widetilde{\mathbf{\Delta} \mathbf{L}} \tilde{\mathbf{I}}_{m-1}(z, \omega), \\
& \frac{d}{d z} \tilde{\mathbf{I}}_{m}(z, \omega) \\
& =-j \omega\left[\begin{array}{ccc}
\mathbf{C}_{0} & & \\
& \ddots & \\
& & \mathbf{C}_{0}
\end{array}\right] \tilde{\mathbf{V}}_{m}(z, \omega)-j \omega \widetilde{\boldsymbol{\Delta} \mathbf{C}} \tilde{\mathbf{V}}_{m-1}(z, \omega) .
\end{aligned}
$$

The above equation is equivalent to an MTL with distributed sources (cfr. [11]). It is important to remark that such distributed sources depend only on the solution at the previous iteration step, and they are therefore known when solving (6) at step $m$. Moreover, the MTL is now described by block diagonal p.u.l. matrices, which allows solving each block independently by considering

$$
\begin{aligned}
\frac{d}{d z} \mathbf{V}_{k, m}(z, \omega) & =-j \omega \mathbf{L}_{0} \mathbf{I}_{k, m}(\omega)+\mathbf{V}_{F k, m}(z, \omega), \\
\frac{d}{d z} \mathbf{I}_{k, m}(z, \omega) & =-j \omega \mathbf{C}_{0} \mathbf{V}_{k, m}(\omega)+\mathbf{I}_{F k, m}(z, \omega)
\end{aligned}
$$

for $k=0, \ldots, 454$, where

$$
\begin{aligned}
\mathbf{V}_{F k, m} & =\left[-j \omega \widetilde{\boldsymbol{\Delta L}}_{k} \tilde{\mathbf{I}}_{k, m-1}(z, \omega)\right]_{k \text { th row block }} \\
\mathbf{I}_{F k, m} & =\left[-j \omega \widetilde{\boldsymbol{\Delta}}_{k} \tilde{\mathbf{V}}_{k, m-1}(z, \omega)\right]_{k \text { th row block }}
\end{aligned}
$$

The problem (6) is solved for increasing $m$ starting from null equivalent sources and updating them with (8), until the solution has converged within a given tolerance $(0.1 \%$ in this paper). Thereafter, the vectors of PCE coefficients $\tilde{\mathbf{V}}$ and $\tilde{\mathbf{I}}$ are obtained as the sum of all the computed perturbation terms.

The new proposed approach requires the solution of $M K$ equivalent MTL problems of size $n$ (i.e., the number of conductors of the original transmission line), with $M$ the total number of perturbation steps and $K$ the number of PCE coefficients. This is opposed to the single simulation of an MTL of size $n K$. Therefore, although the number of perturbations $M$ may vary with the frequency and with the amount of variation in the line parameters, the proposed method is expected to outperform the classical SGM when the number of conductors and/or PCE coefficients is large, as is the case of the shielded cable of Fig. 1, for which $n=7$ and $K=455$.

\section{NUMERICAL RESUltS AND VALIDATION}

In this section, the proposed perturbative technique is compared against the classical SGM implementation in the computation of the mean and standard deviation of some explicative examples of voltages at the terminations of the shielded cable of Fig. 1. The PCE of the p.u.l. inductance and capacitance matrices are taken from [10]. Their augmented counterparts $\tilde{\mathbf{L}}$ and $\tilde{\mathbf{C}}$ are readily obtained. The simulations are performed on a PC with an Intel(R) Core(TM) i7-6700, CPU running at $3.4 \mathrm{GHz}$ and $16 \mathrm{~GB}$ of RAM.

As an example, Fig. 2 illustrates the common mode (CM) voltage at the near end (top left) and the differential mode (DM) voltage at the far end (top right) of the two conductors used for differential signaling, as well as the induced singleended crosstalk voltages on conductors no. 3 at the near end (bottom left) and no. 5 at the far end (bottom right). The comparison between the classical SGM (solid gray lines) and the proposed perturbative SGM (dashed black lines) shows excellent agreement, thus proving the accuracy of the proposed approach.

As far as the computational efficiency is concerned, the perturbative SGM only requires 2463 s to analyze 500 frequency points, in contrast to the 24079 s required by the classical SGM. A speed-up factor of roughly one order of magnitude is thus achieved. This is remarkable when considering that the perturbative approach requires to deal with additional distributed sources, in analogy with the analysis of nonuniform MTLs (cfr. [7]), even for a uniform MTL. For this reason, the proposed method is expected to be even more beneficial for nonuniform MTLs.

It should be mentioned that the time required to compute the PCE of the p.u.l. parameters is excluded from the above analysis, since it is a one-off preliminary step that is common to both SGM-based approaches, and does not need to be reperformed when different terminal configurations and/or cable lengths are considered. 

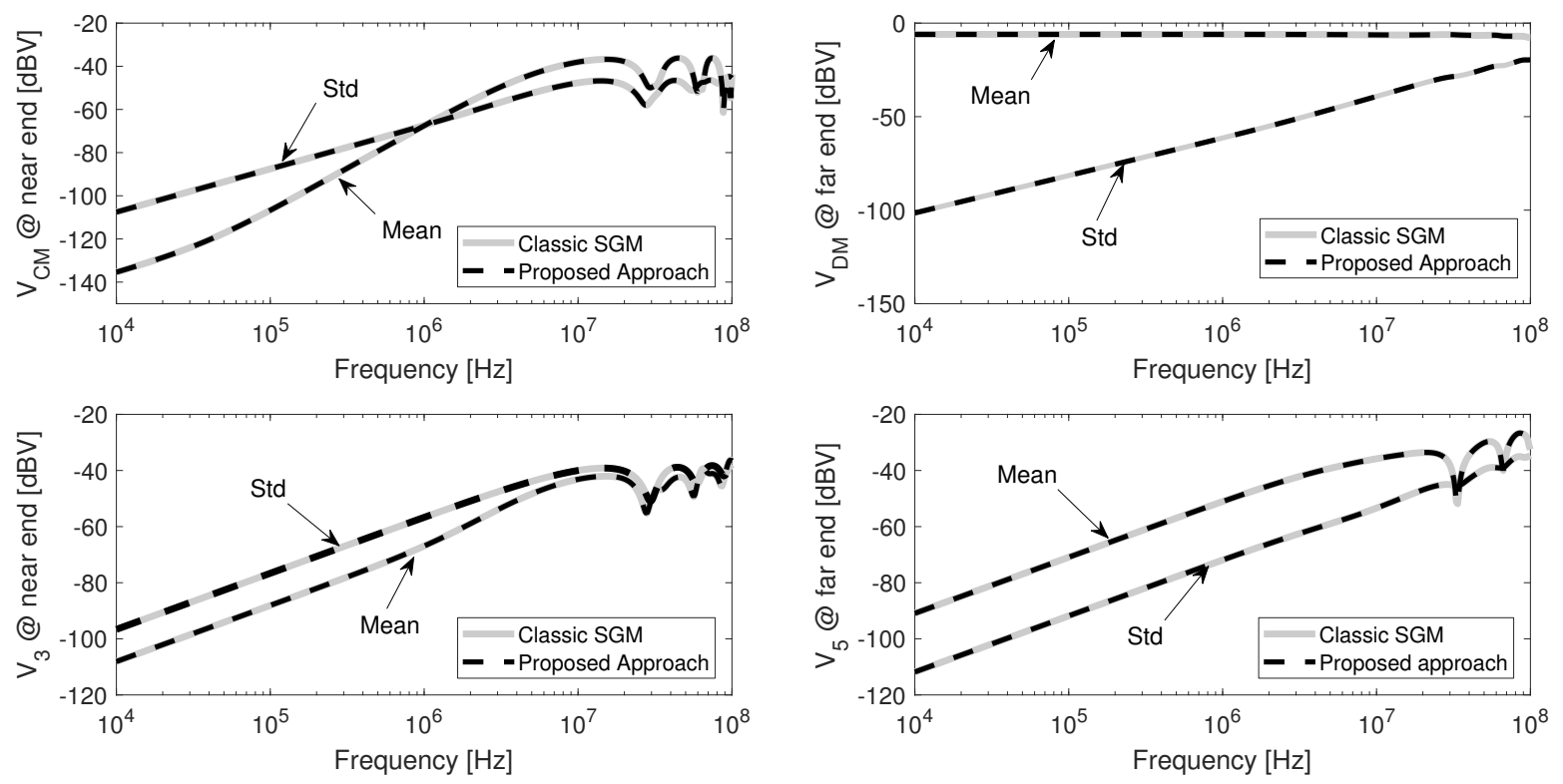

Fig. 2: Mean and standard deviation of (a) DM voltage at far end of wires no. 1 and 2, (b) CM voltage at near end of wires no. 1 and 2, (c) crosstalk voltage at near end of wire no. 3 and (d) crosstalk voltage at far end of wire no. 5 predicted by classical SGM method and proposed approach.

\section{CONCLUSION}

This work presented an hybrid technique for statistical analysis of wiring harnesses affected by several random geometrical parameters. The proposed approach is based on a perturbative reformulation of the traditional SGM, and allows mantaining the size of the MTL networks to be solved equal to the size of the original MTL structure under analysis. According to such a perturbative approach, the first-order PCE coefficient is used to evaluate an initial prediction of relevant voltages/currents. Then this prediction is iteratively refined by including distributed voltage and current sources involving the higher-order PCE coefficients. The proposed approach has been applied for statistical assessment of a shielded cable involving a large number of wires (7), as well as of random parameters (12). With respect to traditional SGM, it has been proven that the proposed approach allows a significant reduction of computational time, while retaining the same prediction accuracy. Finally, it is worth mentioning that, although here applied to uniform MTLs only, the proposed approach looks promising (and even more beneficial) to handle the practical-relevant case of wiring harnesses or interconnects with cross-section randomly varying along the line length.

\section{REFERENCES}

[1] M. Wu, D. G. Beetner, T. H. Hubing, H. Ke, and S. Sun, "Statistical prediction of reasonable worst-case crosstalk in cable bundles," IEEE Trans. Electromagn. Compat., vol. 51, no. 3, pp. 842-851, 2009.

[2] C. Jullien, P. Besnier, M. Dunand, and I. Junqua, "Crosstalk analysis in complex aeronautical bundle," in
Proc. 2013 Int. Symp. on Electromagn. Compat., Bruges, Belgium, Sep. 2013, pp. 253-258.

[3] F. Paladian, P. Bonnet, and S. Lalléchère, "Modeling complex systems for emc applications by considering uncertainties," in Proc. 2011 XXX URSI General Assembly and Scientific Symp., Istanbul, Turkey, Aug. 2011, pp. $1-4$.

[4] G. Spadacini, F. Grassi, and S. A. Pignari, "Field-towire coupling model for the common mode in random bundles of twisted-wire pairs," IEEE Trans. Electromagn. Compat., vol. 57, no. 5, pp. 1246-1254, 2015.

[5] I. S. Stievano, P. Manfredi, and F. G. Canavero, "Stochastic analysis of multiconductor cables and interconnects," IEEE Trans. Electromagn. Compat., vol. 53, no. 2, pp. 501-507, 2011.

[6] P. Manfredi, D. V. Ginste, I. S. Stievano, D. D. Zutter, and F. G. Canavero, "Stochastic transmission line analysis via polynomial chaos methods: an overview," IEEE Electromagn. Compat. Mag., vol. 6, no. 3, pp. 77-84, 2017.

[7] P. Manfredi, D. De Zutter, and D. V. Ginste, "Analysis of nonuniform transmission lines with an iterative and adaptive perturbation technique," IEEE Trans. Electromagn. Compat., vol. 58, no. 3, pp. 859-867, 2016.

[8] X. Wu, F. Grassi, P. Manfredi, and D. V. Ginste, "Perturbative analysis of differential-to-common mode conversion in asymmetric nonuniform interconnects," IEEE Trans. Electromagn. Compat., vol. 60, no. 1, pp. 7-15, 2018.

[9] S.-K. Chang, T. K. Liu, and F. M. Tesche, "Calculation of the per-unit length capacitance matrix for shielded 
insulated wires.” Technical Report, Science Applications Inc. Berkeley Calif, AD-A048 174/7, Tech. Rep., 1977.

[10] P. Manfredi and F. Canavero, "Efficient statistical extraction of the per-unit-length capacitance and inductance matrices of cables with random parameters," Advanced Electromagnetics, vol. 4, no. 1, pp. 22-30, 2015.

[11] C. R. Paul, Analysis of multiconductor transmission lines. John Wiley \& Sons, 2008.

[12] D. Xiu and G. E. Karniadakis, "The Wiener-Askey polynomial chaos for stochastic differential equations," SIAM J. Sci. Comput., vol. 24, no. 2, pp. 619-644, 2002. 